\title{
Efficient Plan for Art Transaction through Non Fongible Token(NFT)
}

\author{
Kyoohoon $\mathrm{Jo}^{1}$, Jeongmin $\mathrm{Ko}^{2}$ \\ ${ }^{1}$ Student, Department of Culture and Arts Management, Hongik University, Korea, \\ jkh660210@naver.com \\ ${ }^{2}$ Professor, Department of Culture and Arts Management, Hongik University, Korea, \\ spin3001@naver.com
}

Corresponding author: Jeongmin Ko

\begin{abstract}
This study is aimed to examine how art works are traded through Non Fongible Token(NFT) that are different from the existing trade methods such as galleries, auctions, and art fairs in art trade. It also analyzes how art trades such as game items are traded through NFT. Furthermore, it is intended to place appropriate values to the works created through NFT, have clear ownership, and avoid hacking when collectors try to buy at NFT exchanges. Lastly, in relation to the environment, is to closely monitor whether or not it causes environmental problems like the existing virtual currency, and to achieve 'efficient art trade' with a more eco-friendly system. By doing so, it is possible to lower the price of works and increase the number of collectors and artists who buy and sell artworks by partially dividing the works of art that have risen in the offline market as the transaction method through NFT, which is different from the existing transaction method, rises in the offline market. This can be seen as a great help to job creation and income increase in the art industry from the industrial point of view. The scope of this research is that what is Blockchain?, what is NFT?, NFT and games, NFT and arts, Interview with 2CEO, NFT and art transactions, advantages and disadvantages of art transactions through NFT, analysis results ,conclusions and limitations. As for the research method, the data were qualitatively investigated and analyzed through interviews with major related companies, literature study and case analysis, and legal review of the supervisory body.
\end{abstract}

Keywords: Blockchain, NFT, Art Trade, Art Works, Collectors, NFT Exchange

\section{Introduction}

\subsection{Research Purpose}

The Korean art market, which was expected to stagnate due to concerns about the pandemic, has also washed away concerns, and the works of famous painters are out of art works at galleries, auctions, and art fairs. However, it is true that the reality of the Korean art market is much lacking compared to the current boom in the art market. This is true in view of the fact that digital art, that is, 'art transaction through Non Fongible Token(NFT)', which is blowing in advanced art countries including the United States, is not yet in the spotlight in Korea. However, considering that Korea's 'virtual currency through blockchain' transactions, which is the same basis as NFTs, are world-class, it is highly probable that art transactions through NFTs will be activated in Korea soon.

NFT is a digital asset made impossible to change, delete, or cancel by giving a separate unique

Received: July 14, 2021; $1^{\text {st }}$ Review Result: August 30, 2021; $2^{\text {nd }}$ Review Result: October 13, 2021 Accepted: November 30, 2021 
recognition value to the digital based on blockchain technology. That is, it is a file with proof of ownership.

When purchasing digital assets such as digital artwork, tweets, GIFs or other digital files, buyers receive NFT tokens. This is a kind of serial number encrypted with the artist's signature. Thus, a token can be viewed as a unique signature or certificate. These transactions are registered on the blockchain (a digital system that records information in a way that makes fraudulent activity nearly impossible) and published digitally. Once published, it cannot be changed, deleted or undone. Thus, buyers of digital assets become the official owners of goods that are available to many people.

Until now, the art transaction was a method in which ownership was transferred through large galleries, auction houses, art fairs, and some cyber transactions. Although it has the advantage of increasing the transaction pie and increasing the stability of the artwork transaction, it is enough to cause great doubts in terms of the appropriateness of the traded artwork price, which may cause confusion in the art market.

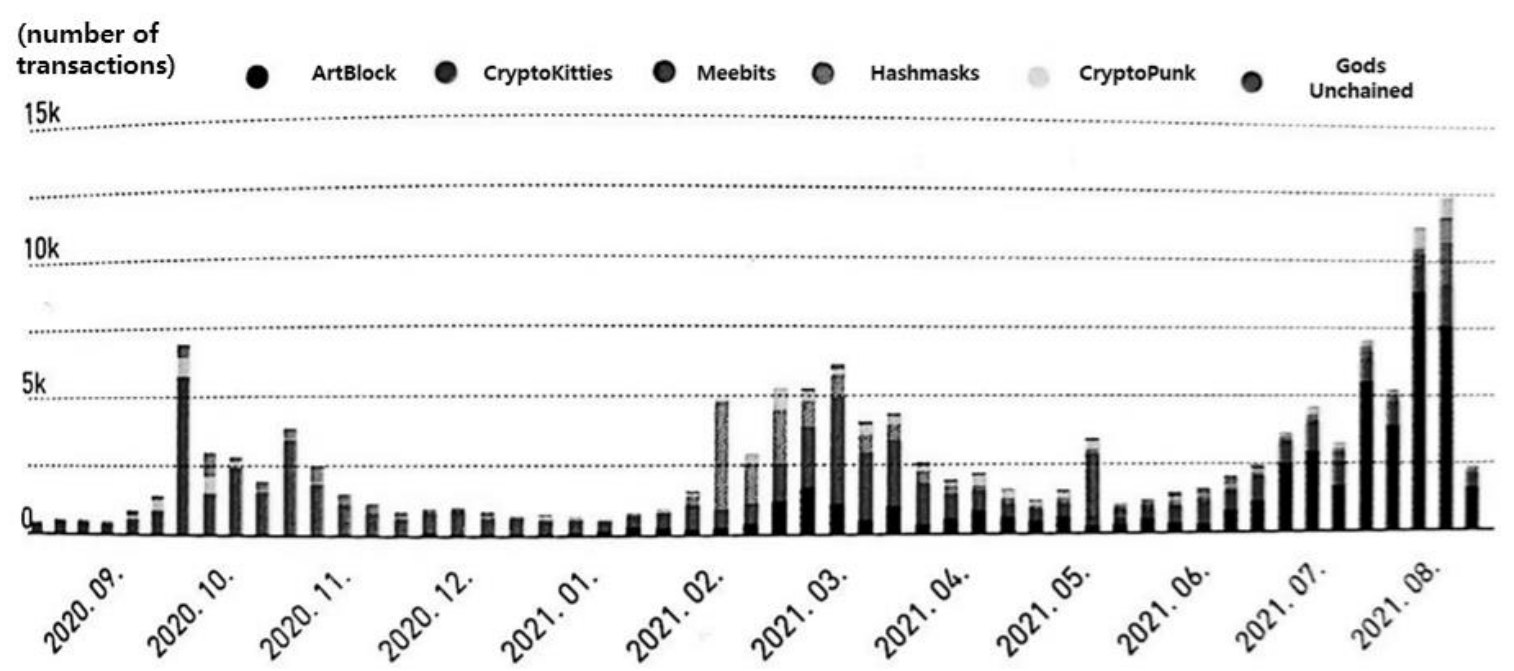

[Fig. 1] Weekly Change in the Number of NFT Secondary Market Trades (https://www.theblockcrypto.com)

Speaking of the 'half cycle' outlined by Gartner, an IT advisory organization [Fig. 1], NFT experienced severe growing pains earlier this year and grew rapidly through positive events[1].

Urrently, the size of the NFT market is growing rapidly. According to NonFungible.com, an NFT market analysis firm, the NFT transaction volume in the first quarter of this year reached \$2 billion (about 2.3 trillion won). The size of the NFT market, which was $\$ 140$ million (about 160 billion won) in 2019 , is growing very rapidly to $\$ 340$ million (about 390 billion won)12) last year. The NFT market is divided into categories such as collectibles, art, metaverse, sports, games, and utilities, and the largest market is collectibles, accounting for $66 \%$ of the transaction volume. After that, $14 \%$ in the arts field, $7 \%$ in the metaverse field, $5 \%$ in the game field, and $1 \%$ in the utility field[2].

The purpose of this research should be the value of works created through NFT which is appropriate and ownership is clear, and should not be hacked when collectors try to buy at NFT exchanges. Lastly, in relation to the environment, is to closely monitor whether or not it causes environmental problems like the existing virtual currency, and to achieve 'efficient art trade' with a more eco-friendly system.

Therefore, it can be seen that researching and analyzing 'art transaction through NFT' could help in 
the scalability and safety of the art market and the suitability of the traded art price. Ultimately, it can be helpful for efficient art transactions.

\subsection{Research Scope and Question}

According to the thesis, 'art transaction through NFT', which is the main research subject, has been traded in a certain part, though not enough to replace offline, for several years at home and abroad. It is not well maintained, so there is still a lot of uncertainty in the transaction.

This thesis is about what blockchain and NFT. It identifies what kind of art transaction is desirable through NFT by analyzing the preceding research on what are the examples of art transactions for domestic and foreign NFTs, and game industries where NFT transactions are established as necessary conditions.

Research questions are as follow. First, what is NFT and art?, Second, through what process is art transaction made through NFT?, Third, what is needed to make art transactions through NFTs efficient?, Fourth, what will be corrected or supplemented in the future other than the existing art transaction through NFT?.

\section{Theoretical Background}

\subsection{Art and Art Market}

\subsubsection{What is Blockchain?}

It is a distributed data storage technology that transparently records transaction details in a ledger that anyone can read, and copies and stores them on multiple computers. Multiple computers verify records to prevent hacking.

It is a data-distributed storage technology that copies and stores data in multiple computers at once by inputting data into blocks and entering them in a chain form. Distributed data storage technology allows users participating in transactions to view data and transaction details, and makes information and data unique to all transaction users so that data cannot be tampered with or hacked.

The characteristic of blockchain is first, it can be distributed and stored. Hacking was possible if only the central server was attacked, but since data is stored by distributing data with several blockchain users, it is difficult to counterfeit and tamper and it is virtually impossible to hack because it has to attack all of the participants' data. Second, there is no need for a central administrator. In financial institutions, etc., authentication and registration were required for a central administrator, but a blockchain that stores and proves a large number of data does not actually require a central administrator. Third, the issuance and distribution of virtual currencies such as Bitcoin, which do not require a central control system, became possible because of the block chain.

\subsubsection{What is NFT?}

Non-Fungible-Token (NFT) is a token that cannot be replaced with other tokens because it has a unique value. Cryptocurrencies such as Ripple (XRP), Bitcoin (BTC), and Ethereum (ETH), which we have heard a lot about, are fungible tokens that do not distinguish individual tokens. Since each token has the same properties, it can be traded or exchanged with each other. An NFT, on the other hand, can be likened to a performance ticket with details such as the buyer's name, date, and seat. The NFT can implement and commercialize tangible and intangible assets with intrinsic value in the digital world through blockchain technology. Since details of assets such as transaction history and owner are safely 
stored in the blockchain, where it is difficult to falsify data, the authenticity and scarcity of digital assets can be guaranteed and ownership of the assets can be proven.

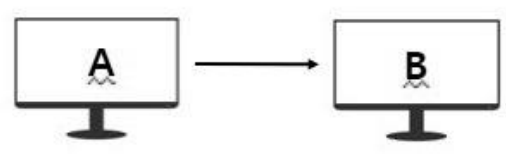

$A$ requests $B$ for transactions
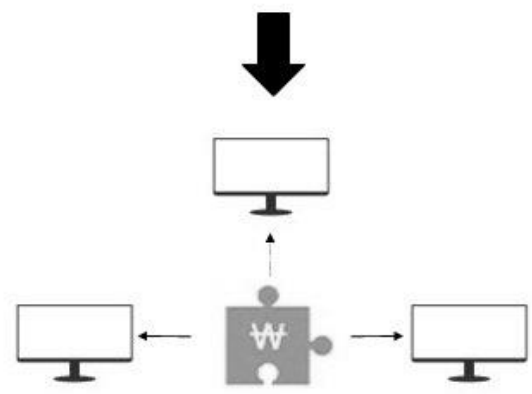

Transaction blocks are created and transmitted to all participants in the network.

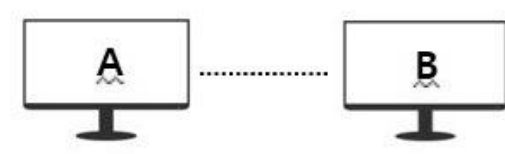

Transaction completed
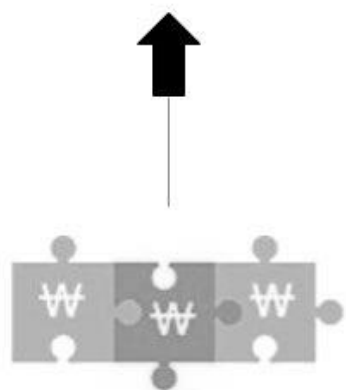

Blocks in which information mutual verification has been completed are combined with chains.

[Fig. 2] Blockchain Flow[3]

It can identify and track digital assets, directly connect issuers and users without physical constraints, and enable efficient transactions and circulation. As the demand for scarce assets arises, a trading market is formed and its value rises. Since scarce assets can increase in value over time, interest in NFT investments is growing recently. Transactions through NFTs, where assets are digitally proven to have ownership and scarcity, will continue to be active in the future, and growth will continue to increase in the future society.

NFTs can be easily exchanged for unique digital assets. It is created on a blockchain and is mainly based on Ethereum, and various other blockchains such as Wax, Matic, and Flow. The concept of NFT has been around since 2015, but it started to surface through the first project created on the Ethereum blockchain through the ERC20 standard in 2017. Over the next two years, many NFT standards have been accepted and used.

The main difference between NFTs and regular cryptocurrencies is that NFTs are not directly compatible with other assets based on their ID, value, and utility. Every NFT consists of metadata that gives a token its individuality. Metadata may include size, name, sparse value, and the like.

Non-fungible Token, literally, is a form of digital ownership that certifies that a particular piece of content can be copied and reproduced as the unique property. This method not only helps solve the problems of counterfeiting and tampering that existed in existing works of art, but also helps to solve the problem of digital copying and reproduction. It is expected to increase the overall value of the content[4].

NFTs not only exist in the digital space, but can represent any type of physical asset. It serves as a kind of 'digital twin' of everything that exists in the real world, enabling ownership and trading of physical possessions in digital marketplaces. True ownership is one of the key determinants of an NFT. As the digital economy continues to develop, NFTs will play a pivotal role in bringing the digital and physical worlds closer than ever before. 


\section{Prior Research}

\subsection{NFTs and Games}

\subsubsection{Study Framework}

The first component of the research model is the social and economic impact of a new innovation factor. The value and adaptability factors proposed in the VARIM framework of Afuah (2017) are the socio-economic value of the innovation and the company. It means appropriate response capability, and it can be defined as a socio-economic factor created by applying blockchain NFT technology to game development.

The second component is the impact of innovation factors on the industrial ecosystem. Among the 10 types of innovation proposed by Keeley et al. (2013), network capability and product system capability are defined as industrial ecosystem impact. On one hand, this means the impact that the game industry ecosystem centered on the existing business model will have due to the new innovation factor. The third is the influence on the differentiation of the company concerned. This is a concept including scarcity and non-imitation proposed by Afuah (2017), but rather than implying permanent scarcity or impossibility due to the universal concept of blockchain technology itself, it is included in the proposal of Keeley et al. (2013). It can be defined as the types of brand differentiation, service differentiation, and product performance differentiation through established, leading position. In other words, it refers to the influence that a company has to differentiate and position within the industry. The fourth is customer benefit impact, which means the overall value-added effect that customers will enjoy through the new innovation factor. Customer benefit includes the value suggested by Afuah (2017), and in Keeley et al. (2013) model, which means the benefit effect of customers through customer participation and service included in the customer experience area.

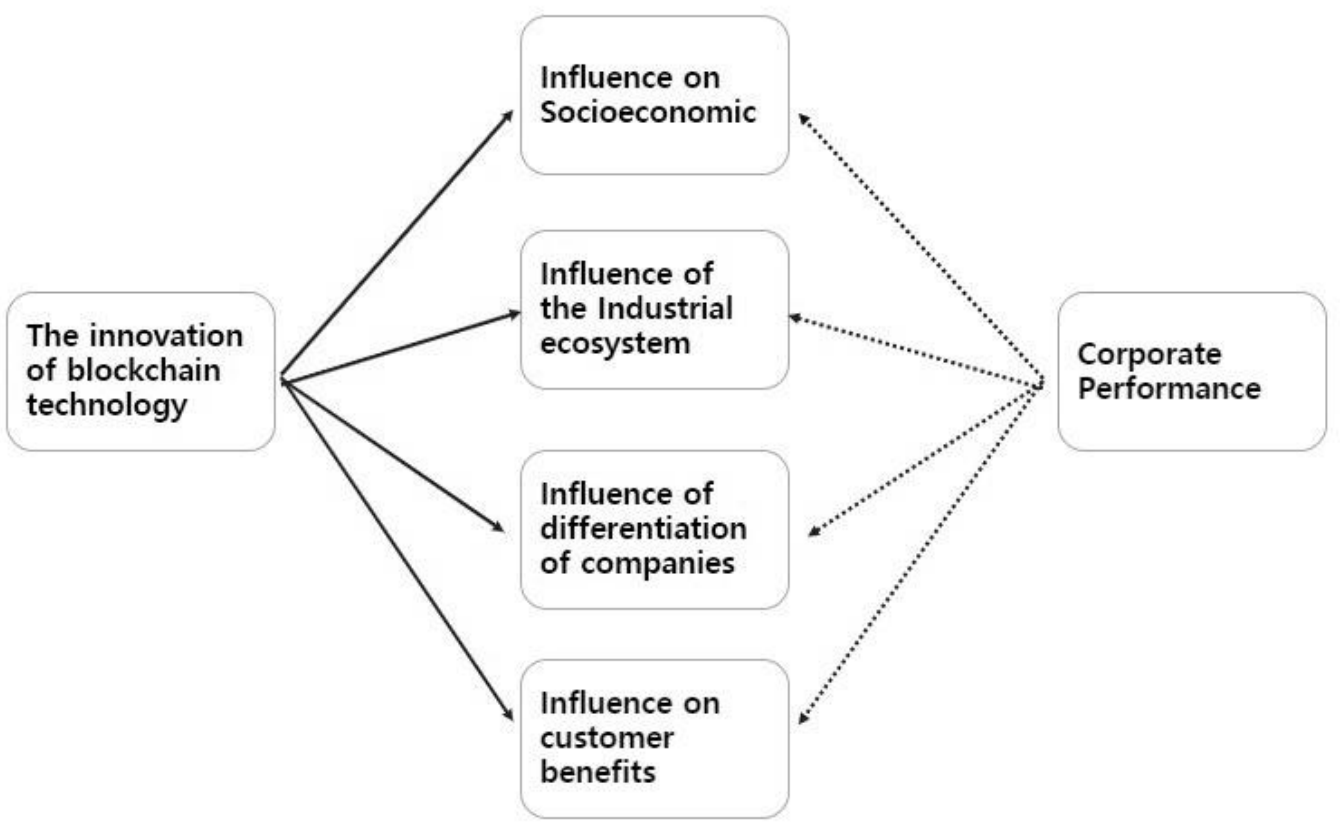

[Fig. 3] Research Model

\subsubsection{Conversion Flow between 'In Game Item' and 'NFT'}

When users select an item to change to NFT in-game, they are connected to the blockchain mainnet wallet link page, create an NFT, and then send the NFT item to the wallet. After that, the 
corresponding item information is deleted from the in-game DB table. Conversely, when changing an NFT item to an in-game item, the item in the blockchain mainnet wallet is deleted and the corresponding item information is added in the in-game DB table. At this time, each conversion record is left in the game DB and the history is saved[5].

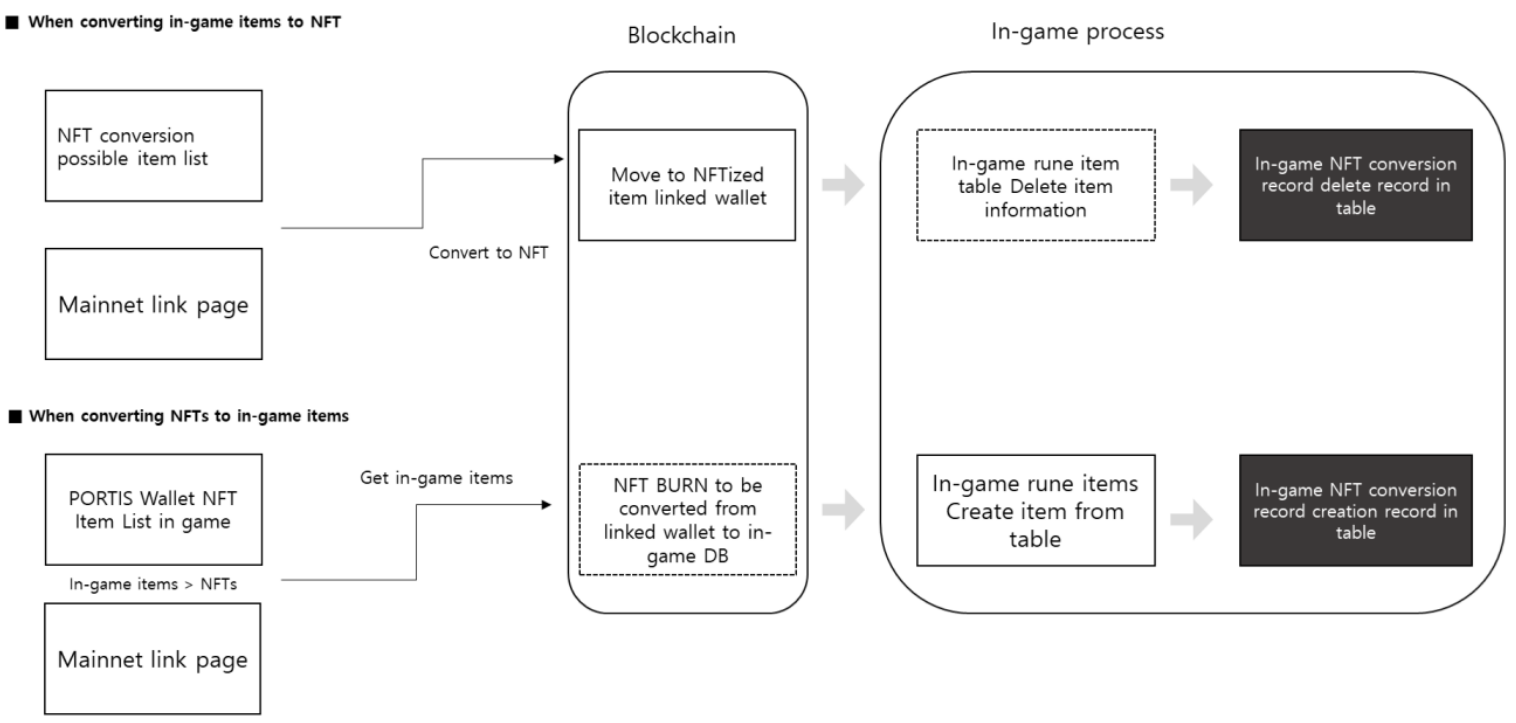

[Fig. 4] User Flow related to Item NFT Conversation[6]

\subsection{NFT and Arts}

\subsubsection{The Concept of NFT and Arts}

NFT is a unique proof of ownership based on Ethereum that aims for decentralized value. Issues a certificate and guarantees digital content transaction through the transaction of the certificate. These NFT technologies NFT art is what has been grafted onto digital art. NFT Art is a blockchain-based digital content It is a media blockchain that deals with NFT art is a digital art of Ethereum's decentralized nature. We aim for the decentralized ideal of the media block chain by grafting it on the platform[7].

\subsubsection{Framework of Trade}

The framework of nft transaction consists of the procedure shown in [Fig. 4], and related key terms are shown in [Table 1] below.

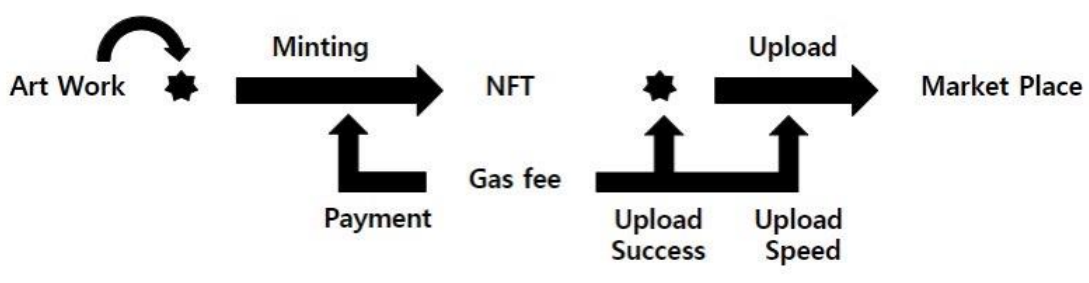

[Fig. 5] Framework of NFT 
[Table 1] NFT Key Concepts[8]

\begin{tabular}{|c|c|}
\hline Minting & Making digital works into NFTs through the Ethereum blockchain. \\
\hline Gas & $\begin{array}{r}\text { Artists who want to sell their art work in NFT must upload their work to } \\
\text { the marketplace, and gas minting fee is incurred. Also the upload speed } \\
\text { is different. }\end{array}$ \\
\hline Transaction & $\begin{array}{r}\text { Sending tokens from the Ethereum system to the network or sending Ethereum } \\
\text { to another place. Linking the artwork with the NFT is also part of the } \\
\text { transaction and you will have to pay a fee even if the transfer fails. }\end{array}$ \\
\hline Metadata & $\begin{array}{c}\text { Data representing other materials. } \\
\text { NFT does not include all other materials, but only the metadata of the work. }\end{array}$ \\
\hline
\end{tabular}

\subsubsection{Transaction Flow}

First, the artist needs to buy Ethereum, a cryptocurrency. A cyptocurrency can be bought by dividing it by 0.1 and 0.5 .

Second, install Metamask (virtual currency wallet). Metamask is one's own wallet for storing Ethereum. A lost password cannot be retrieved.

Third, the exchange transfers Ethereum to MetaMask. A fee should be paid to the exchange, thus, one will transfer Ethereum to the exchange using MetaMask.

Fourth, access the exchange. After the account connection is complete, the artist should create a store and prepare to register NFT works in the store.

Fifth, register and sell works.

\section{The Methods Used in the Study}

\subsection{Interview}

\subsubsection{Interview with CEO Chang Hyun Guen of Eco Capital on November 9,2021}

First, what does it mean to buy NFT?

Currently, it is one of the means of investment through digital art, but if display technology develops rapidly in the future, NFT can be a useful object of appreciation.

Second, how is collecting NFTs different from collecting works of art in the traditional art market?

NFT is different from existing real art collections, where the purpose of appreciation and enjoyment is strong, because NFT is more financially inclined than pure appreciation and enjoyment.

Third, what is the biggest threat when collecting NFT works?

NFT itself has perfect security because it cannot be copied, but the loss of the device to store it, or forgetting the access password is a threat. Also, there is a limit to the display technology that can be enjoyed.

\subsubsection{Interview with Director Oh Se Gun of Seoul Auction Blue on November 12, 2021}

First, do you think that the value of NFT works is different from existing real works in some ways.? since the movement path such as who sells and buys is recorded on the block chain, transaction traces are left and anyone can see them, so it is easy to distinguish genuine products from imitations unlike real works. In other words, it is like a real estate registration certificate. 
Second, in order to generally settle it for the purchase procedure with NFT it is traded with virtual currency (Ethereum).

dose it cost a lot more money to buy art? (Most of them will purchase Ethereum in advance and have it.)

nowadays, some cryptocurrencies other than Ethereum are also commercialized, but NFTs are still mainly traded with Ethereum. Although Ethereum is less volatile than other cryptocurrencies due to NFT, the price of NFT works also fluctuates according to changes in Ethereum, although not as much as the volatility of Ethereum's price.

Third, what are the security considerations when buying and selling artworks with NFT?

personal wallet SEED Classification 16-word accounts should be managed like house documents.

Fourth, please explain the various existing NFT-related platforms (marketplaces).

In OPEN SEA, NFT-related items in various fields such as art works, game items, sports collectibles and real estate are traded. On the other hand, SUPER RARE and NIFTY GATE WAY are mainly traded on works by famous artists.

Fifth, since Seoul Auction blue is a subsidiary of Seoul Auction, if NFT art works are hosted by Seoul Auction, the process will be different from the general offline auction process. How does it work?

Taking the beeple work as an example, proceed as follows.

-Beeple creates works in the form of digital image files

-Generated by encrypting a unique hash value that identifies the file

-Generate metadata for works with hashes

-Upload works and metadata to IPFS (Inter Planetary File System)-based storage, a decentralized distributed storage technology

-Mining the NFT of the work with Maker Token V2 that follows the Ethereum ERC-721 protocol

-Seoul Auction puts mined NFTs in online auction

-The successful bidder transfers the Ethereum corresponding to the successful bid price to the Seoul Auction's cryptocurrency.

-Beeple sends NFT tokens to the winning bidder's stake and receives the winning bid price

Sixth, if an existing famous artist's work is produced as a digital file NFT works, what is the procedure? For example, "Park Soo-geun's Laundry Ground" was going to be released on NFT, but was canceled because it did not go through the consent of the original author?

NFT works basically require the consent of the original author. If the owner of the NFT work transforms and sells the original work without the consent of the original work, it becomes a imitation work. However, the owner of the original work of the original author is free to sell the work.

Lastly, what do you think is the future prospect of NFT art?

The world of NFT art will grow infinitely in the future. Of course, it will take some time and coexist with real works, but after a certain amount of time, the existing real art market will change to NFT works with only a certain part remaining.

\subsection{Case Analysis}

\subsubsection{NFT and Art Transactions}

The NFT took its place early in the arts sector. In a situation where physical contact is difficult due to COVID-19, NFT provides an opportunity for artists to directly sell their work, and an opportunity for users to touch and own their work.

In October 2020, for the first time, Christie's, a world-class art auction house, auctioned off a blockchain-based work 'Portrait of a Mind'. It is a work of 40 paintings, and NFT is grafted into the core of 
each painting to disperse ownership. The work sold for $\$ 131,250$ (about 144.6 million won), ten times higher than the expected price.

At an NFT auction held by Christie on March 11, 2021, Beeple's 'Everydays-The First 5000 Days' was sold for $\$ 69.34$ million, the highest price among NFT artworks. This work is a collage work created by collecting photos that Bipple has posted online every day since 2007 . He has worked with pop stars such as Justin Bieber and Katy Perry.

The 10-second video 'CROSSROAD' produced by Beeple was sold for $\$ 67,000$ (about 75 million won) in 2020.

It was sold and sold back for $\$ 6.6$ million (about 7.3 billion won) in February 2021. 'The First Ever Edition Of Rick And Morty Cryptoart' from the crypto art collection 'The best I could Do' released by Justin Roiland, co-creator of the animated series Rick and Morty, released on January 19, 2021 for $\$ 150,000$ (about 160 million won) was sold. On the NFT marketplace Nifty Gateway, in December 2020, Star Wars-themed NFT works were traded for $\$ 777,777$ (about 850 million won). Digital artist Trevor Jones' animated work 'Picasso's Bull' sold for $\$ 19,500$ (about 20 million won). On March 18, 2021, Mari Kim's NFT work 'Missing and found (2021)' was sold for the first time in Korea. It is the first art work in Korea to which NFT is applied, and contains a female character with big eyes called 'eyedoll'. It started at 50 million won and sold for about 600 million won (288 ETH).

\section{Analysis Results}

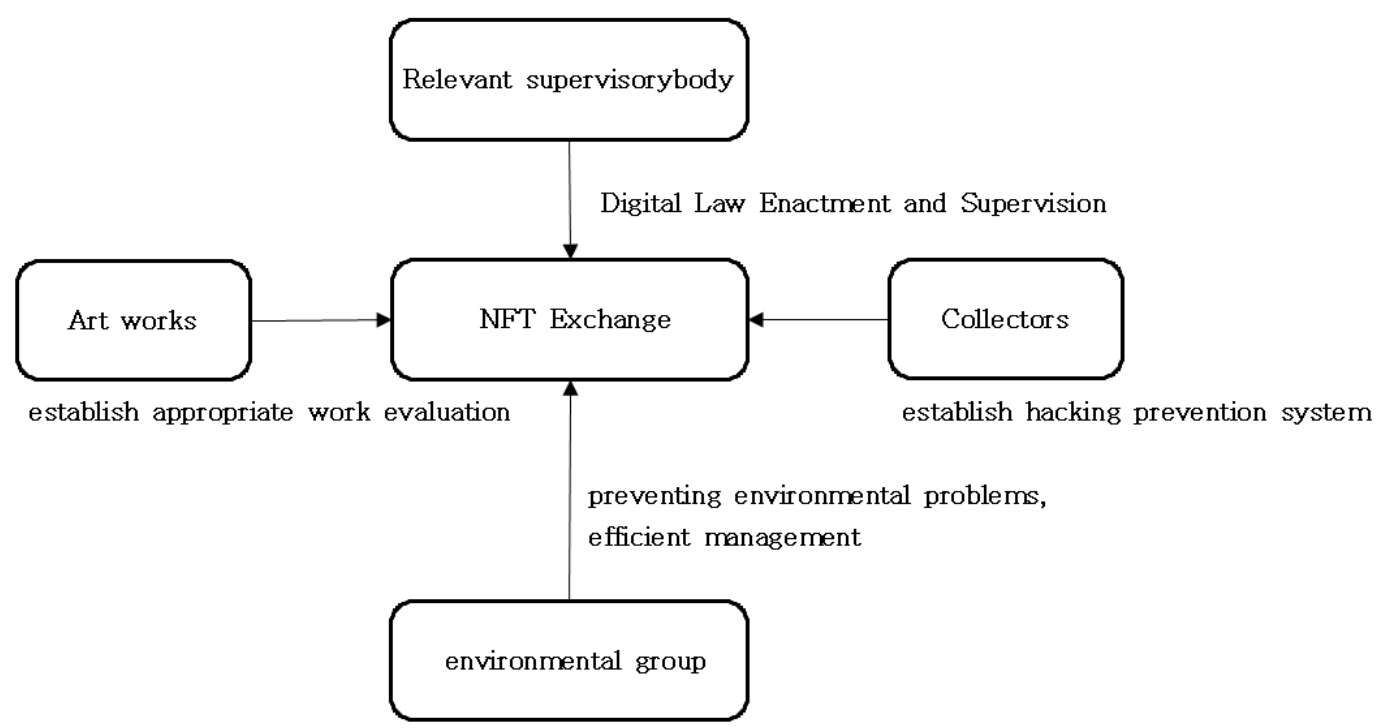

[Fig. 6] NFT Art Trade Analysis

\subsection{First, the Importance of the Exchange}

A certain fee is paid when money is converted into Ether or other coins to buy works on the NFT exchange, or when the works are uploaded to sell. Since the money has been paid, the exchange has to take responsibility for it. Even if the system is not in place right now, there is no guarantee that the problems that occurred in the cryptocurrency exchange will not occur in the NFT exchange.

\subsection{Second, the Value of NFT Works}

Although it is a subtle and difficult matter, before a digital work can be registered on the NFT 
exchange, it should be possible to notarize whether it is a work of real value or not a precisely crafted counterfeit. The places that can play this role are professional judges at Sotheby's, Christie's, and leading investment banks, and in Korea, Seoul Auction, K Auction, and art appraisal groups.

\subsection{Third, NFT-related Laws and Regulations are Newly Established}

Digital-related laws should be revised and newly established in order to prevent plagiarism, hacking, illegal planning of stock trading in advance, and regulate price increase.

\subsection{Fourth, it is to Solve Environmental Problems}

Blockchains using proof-of-work (PoW) such as Bitcoin and Ethereum consume huge amounts of power and have a negative impact on the environment. Although NFT is mainly done through 'Ethereum', Ripple's own blockchain recently claims that "XRPL consumes very little energy to process transactions and is 120,000 times more efficient than proof-of-work.[9]" Efforts are needed to newly integrate a system equipped with a system with high power efficiency to the art trade.

\section{Advantages and Disadvantages of Art Transactions through NFT}

[Table 2] Advantages and Disadvantages of Art Transactions through NFT

\begin{tabular}{|c|c|c|}
\hline division & Advantages & disadvantage \\
\hline Utility & scarcity guarantee & Plagiarism Controversy \\
\hline Certainty & easy to track & Ownership Controversy \\
\hline Safety & NFT itself cannot be hacked & Exchange and personal account hacking \\
\hline Expandability & $\begin{array}{l}\text { Acknowledgment of ownership } \\
\text { of parts }\end{array}$ & To illegally plan stock trading in advance. \\
\hline
\end{tabular}

First, the NFT technology ensures that no one can copy or transfer the NFT without the permission of the owner. That is, scarcity guaranteed. But we cannot control who was the first to work on a piece with NFT. Controversy over plagiarism may ensue. In fact, Swedish illustrator Simon Stålenhag has never published NFT works himself and has never given permission to anyone, but found that one of his works is a kind of NFT 'MarbleCard'. There is also an issue on the current NFT's lack of regulation or supervision. This also suggests that the number of cases in which the copyright of the creator is not protected may increase.

Second, NFT has the advantage of being easy to trace because one unique code is assigned to one digital file work. However, if a famous artist's work is arbitrarily converted into a digital file and traded in the NFT, there may be disputes over the ownership, and this work cannot be easily removed from the exchange. This is because NFT is a basic concept of decentralization based on blockchain.

Third, as with coin trading, it can be doubted whether 'legal exchange management' can be possible when NFT is activated. NFT itself has the advantage that it is impossible to hack, but it has the disadvantage that it is difficult to prevent illegal activities of exchanges and hacking of personal accounts.

Fourth, it has the advantage of being able to increase the pie of the entire work of art by 'recognizing ownership of the part' by partially digitizing one's work and putting it in a file, but it is problematic in the adequacy of the transaction price. Of course, there have been constant doubts as to 
whether it is appropriate to pay 10 billion won per picture for existing offline transactions.

"However, NFT is still only a small niche and its applications are very limited. Currently, NFT applications are mainly focused on digital collections, works of art, games and virtual worlds, but in practice NFT can be applied to a wider range of fields. For example, physical assets such as houses can also be tokenized with NFTs. It can also be used in financial markets such as asset circulation. Alternatively, it can be used to verify identity, birth certificate, driver's license, educational background, etc. These documents can be safely stored in a digital format to prevent theft or forgery. It can also be used for various tickets such as concert tickets, movie tickets, drama tickets, etc. With NFT, seat numbers and dates can be displayed so that forgery is impossible.[10]"

\section{Efficient Way}

\subsection{A Young Artist's Point of View}

It is necessary to create a website that can promote one's work and actively inform fans of the work through SNS[11].

\subsection{The Art Collector's Point of View}

Storing works purchased through nft in the metaverse can also be an important method. If each collector builds his or her own house on the metaverse and displays the work in a real house, it can be easily stored and resold.

\subsection{A Big Auctioneer's Point of View}

If it is verified that the work is not a forgery or if it is a work with the consent of the original author before it is registered on the exchange, the efficiency of the transaction will inevitably increase.

\subsection{Regulator's Point of View}

The government enacts related digital regulations, and the exchange needs to create a function that can search the works on the exchange through the DB. In this way, counterfeiting disappears and transaction efficiency can be increased.

\section{Conclusions and Limitations}

NFT is expected to continue its growth by combining with new trends such as the metaverse. Metaverse is a compound word of 'meta' meaning abstraction and 'universe' meaning the real world, and refers to the digital transformation of real space. NFT-related industries include game items, artworks, sports collectibles, music records, and real estate. Everything is connected to the metaverse, but especially game items, artworks, and real estate use the metaverse, and the development of technology in this field will open up a new world. The offline art market also seems to be stagnant due to COVID-19, but contrary to expectations, a boom is taking place around the world. However, fields based on virtual reality, such as Metaverse, are not yet booming compared to games and coin fields. It is possible to lower the price of artworks by partially dividing the artworks that have risen in the offline market into digital files, and it can also increase the number of collectors and artists who buy and sell. This can be seen as a great help to job creation and income expansion in the art field from an industrial point of view. 
NFT is a product that combines virtual and reality, and when compared to other applications based on blockchain technology, it feels practical and close to reality. Of course, it is also undeniable that the price of NFT artworks that are traded too high is unrealistic and makes no sense. However, it should also be recognized that NFT has opened a new chapter in the field of digital art and acts as a medium between the virtual world and the real world.

However, there are some limitations to NFT trading, so caution is required.

First, it may cause a plagiarism controversy. If someone copies a work posted on an exchange and creates a file without asking the original author's permission, the exchange may delete it immediately, but there are also cases where it is impossible. This is because, since it is a decentralized platform, it is sometimes impossible to arbitrarily delete it from the exchange.

Second, it is the ownership of the offline original. In the case of art, not only the copyright but also the authenticity of whether the original is digitally created as it is must be checked.

Third, there is the problem of hacking. Since NFTs are based on blockchain, there is no problem with hacking, but exchanges do not. In other words there may be hacking of personal accounts participating in the exchange. The exchange itself has not been a problem so far, but the problem of personal account theft occurs frequently.

Fourth, it can lead to illegally planning stock trading. It refers to the act of inducing the same person to conclude a contract at a high price to raise the price of the product they sell. For example, the person who won the bid for Beeple's work was the founder of the NFT fund Metaverse, and the person who bought Jack Dorsey's tweet for $\$ 2.5$ million was the CEO of BridgeOracle (a virtual currency company).

In conclusion, this study suggested a way to solve the plagiarism controversy, hacking problem, and illegal problem in advance. In the future, we should focus on expanding the NFT area and risk management. The NFT transaction limited to games and art fields can be expanded to music, sports and digital real estate fields. In doing so, problems may arise in which the interests of the participants are conflicting in new areas that laws and regulations do not reach. To overcome this, existing laws must be amended, and new laws in the digital field must be enacted.

Copyright, the right to create derivative works based on the work, the right to distribute the work to the public, the right to publicly perform the work, the right to publicly display the work. Currently, most of the above 5 rights to NFT are owned by the copyright owner, and it does not apply to NFT buyers. However, in the future, buyers are expected to be able to freely trade in the market with various rights.

\section{References}

[1] Seong-Sorah, Rolf-Hoef, Scott-Mclaughlin, NFT Revolution, The Quest, (2021)

[2] Min-Jung Kim, Jae-Woo Park, A Study on Digital M\&L profit creation for Korean Animation using NFT Technology, The Korean journal of animation, (2021), Vol.17, No.3, pp.7-25, DOI: https://doi.org/10.51467/ASKO. 2021.09.17.3.7

[3] https://m.term.naver.com/43667, May 14 (2018)

[4] Connectinglab, Blockchain Trend 2022 -2023, Business Books, (2021)

[5] Sung-Wone Choi, Sung-Mok Lee, Joong-Eon Koh, Hyun-Ji Kim, Jeong-Soo Kim, A Study on the elements of business model innovation of non-fungible token blockchain game : based on 'PlayDapp' case, an in-game digital asset distribution platform, Journal of Korea Game Society, (2021), Vol.21, No.2, pp.123-138, DOI: https://doi.org/10.7583/JKGS.2021.21.2.123

[6] Myoun-Jae Lee, The Development of Blockchain Games by Analyzing the Current status, Journal of Digital Art 
Engineering\&Mutimedia, (2020), Vol.7, No.1, pp.35-47, DOI: 10.29056/jdaem.2020.03.04

[7] Dae-Min Park, NFT Art: Decentralization of the Art World and Aura of Traces, Korean journal of communication\&information, (2021), Vol.109, pp.127-152, DOI: https://doi.org/10.46407/kjci.2021.10.109.127

[8] https://blog.naver.com/cglawfirm/222422318404, Jul 6 (2021)

[9] https://www.tokenpost.kr/article-61917, May 26 (2021)

[10] https://blog.naver.com/smartnari/222371608066, Jul 6 (2021)

[11] Im-Bok Lee, Metabus, The future that already begun Forest of heaven, ISBN-13: 9791188348862, Thousand Tree Forest, (2021) 